\title{
Prevalencia de trastorno de ansiedad en Adolescentes estudiantes de secundaria en el municipio de Aguascalientes
}

Hernández-Sánchez LC, Arellano-Sotelo HA, Martínez-Sandoval JP, Cardona- Mejía CE, GonzálezGutiérrez JL, Guirao-Vega YE, Aguayo-González M, García-Huízar MP, Hernández- Maldonado E, Rosas Cabral A,

\footnotetext{
Resumen

- Los trastornos de ansiedad constituyen una problemática frecuente entre los adolescentes y jóvenes que se - ha incrementado en las últimas décadas. El presente - estudio se efectuó con el objetivo de conocer la fre- cuencia de los trastornos de ansiedad en adolescentes - de 12 a 17 años, estudiantes en escuelas secundarias - de Aguascalientes. Material y métodos: se realizó un - estudio observacional, descriptivo y transversal a partir - de un universo de 13,415 sujetos de entre 12 y 17 - años del Municipio de Aguascalientes. El muestreo fue - aleatorio, sin reemplazo, por conglomerados, bietápico.

- El instrumento de estudio utilizado fue la escala de

- Hamilton para ansiedad validada para México. El aná-

- lisis se realizó mediante estadística descriptiva. Resul-

- tados: quedaron incluidos 416 adolescentes de ambos

- sexos entre 12 y 17 años. De éstos, 108 presentaron

- una puntuación > 16, que considera la existencia de

- un cuadro ansioso. La prevalencia de trastornos de an-

- siedad fue de $25.96 \%$ para este grupo de edad. La

- prevalencia de ansiedad según sexo fue de $36.6 \%$ en - mujeres y de $18.4 \%$ en hombres.
}

\section{Abstract}

Introduction: Anxiety disorder it constitutes a frequent problematic, between the adolescents has been increased in the last decades. The present study with the purpose to analyse the frequency of the disorders of anxiety in adolescents of between 12 o 17 years in Aguascalientes. Materials and methods: We realize an observational, descriptive, transversal and comparative study within a population of 13,415, the selection of the sample was random, and without replace, by conglomerates, in two stages. Our instrument of study was the Hamilton's scale for anxiety approved to Mexico. Results: The sample was 416 students of both sex between 12 and 17 year old, of this 108 showed a score $>16$ and it is considered the anxiety state, with this dates we calculated a prevalence of $25.96 \%$ of anxiety disorder. Conclusions: The prevalence of anxiety by sex was $36.6 \%$ in women and $18.4 \%$ in men.

Palabras clave: Prevalencia, Trastorno de Ansiedad, Adolescentes, Aguascalientes.

Departamento de Medicina. Centro de Ciencias Biomédicas. Universidad Autónoma de Aguascalientes. 


\section{Introducción}

Los trastornos de ansiedad son uno de los diagnósticos psiquiátricos mas frecuentes en la adolescencia ${ }^{1}$ En este periodo de la vida se producen importantes cambios biológicos, que trascienden al área psicológico y social; destaca por su importancia la influencia ambiental y sociocultural que pueden incrementar la frecuencia de este trastorno en todas sus variaciones ${ }^{2}$. El manual diagnóstico y estadístico de los trastornos mentales en su cuarta edición (DSM IV) crea un apartado de clasificación para los trastornos de ansiedad en niños y adolescentes y define ansiedad como un "trastorno que provoca pensamientos preocupantes e injustificados constantes y situaciones de estrés con respecto a actividades cotidianas rutinarias durante varios meses" 3 . De acuerdo a un reporte, en Estados Unidos, entre 1952 y 1993, se observó un incremento de estos trastornos en un $20 \%{ }^{4}$.

Los síntomas que ayudan a detectar los trastornos de ansiedad en los adolescentes son: la inseguridad, el temor, la preocupación, los pensamientos negativos y el temor anticipado ante hechos que se viven como de "difícil tránsito". A veces se acompaña de síntomas somáticos: cardiovasculares, taquicardia, hipertensión arterial, sensaciones de falta de aire, dificultades respiratorias y gastrointestinales, tales como dolores abdominales, cólicos, sequedad en la boca, transpiración profusa, cefalea y contracturas musculares ${ }^{5}$.

Las causas por las cuales se presenta este trastorno en la adolescencia pueden ser desde problemas médicos, como por ejemplo en pacientes con asma, quienes experimentan mas crisis de ansiedad y datos depresivos, en comparación con los que no la padecen ${ }^{6}$. Otras causas como el abuso sexual y la violencia intrafamiliar, pueden condicionar un incremento en los trastornos de ansiedad. Vazsonyi et al observaron a 7,291 adolescentes de Hungría, EUA, Suiza, Holanda y encontraron que la ansiedad aumentaba con la ausencia de soporte y aprobación de los familiares, que es más frecuente en el sexo femenino, así como cuando existe el antecedente depresivo en la madre y es mayor en pacientes que provienen de bajo nivel socioeconómico?

Entre los estudios sobre la frecuencia de los trastornos de ansiedad, se destaca uno efectuado en USA, que refiere que el diagnóstico de ansiedad es del $11.3 \%$ con un rango entre $5.4 \%$ a $17.2 \%{ }^{8}$. Así mismo, se ha reportado que la prevalencia de los trastornos de ansiedad, es una a dos veces más elevada en Estados Unidos que en Europa'.

En otra referencia de Perú, encontraron a los trastornos de ansiedad como la primera causa de consulta, entre los adolescentes, con un $19 \%$, seguido por la esquizofrenia $(18 \%)$, trastorno de comportamiento $(9 \%)$, trastorno depresivo $(8 \%)$ y trastorno de la conducta alimentaría $(4 \%)^{10}$.

En nuestro país, según resultados del cuestionario breve de tamiza- 
je y diagnóstico de problemas de salud mental en niños y adolescentes en la ciudad de México, reportaron que la prevalencia para los trastornos de ansiedad ocupó el tercer lugar como diagnóstico con una frecuencia del $8.2 \%{ }^{11}$.También se encontró diferencia en la prevalencia del trastorno de ansiedad, con predominio del sexo femenino con un $16.5 \%$ y $9.37 \%$ para el sexo masculino ${ }^{12}$. El objetivo de este trabajo fue conocer la prevalencia del trastorno de ansiedad entre los adolescentes del municipio de Aguascalientes.

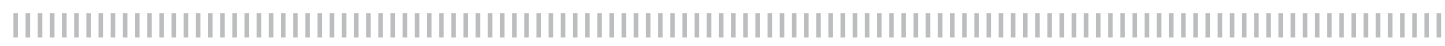

\section{Material y métodos}

Se realizó un estudio observacional, descriptivo, transversal, en los estudiantes de 3er grado de secundaria de las escuelas públicas y privadas del municipio de Aguascalientes de ambos sexos entre 12 y 17 años. Nuestro instrumento de estudio fue la Escala de Hamilton para Ansiedad validada para México, calificativa y autoaplicable. El muestreo fue probabilístico, aleatorio, sin remplazo, por conglomerados, bietápico. Se elaboró un marco muestral, con la lista de grupos proporcionada por el Instituto de Educación del estado de Aguascalientes (IEA) incluyendo secundarias del sector público y privado de este municipio. Para el cálculo de la muestra, se consideró un error máximo estimado del 5\% y una confian- za del $95 \%$, con una tasa de respuesta del $90 \%$ y una tasa de no respuesta del $10 \%$, con lo cual se obtuvo una muestra de 15 grupos de $3 e r$ grado de secundaria, los cuales se seleccionaron mediante muestreo aleatorio simple a partir del marco muestral previamente diseñado, constituyendo la misma 416 alumnos.

El instrumento utilizado se aplicó a todos los adolescentes incluidos en la muestra. Así mismo se evaluó la escala para determinar el puntaje según la intensidad de los síntomas y la observación durante la aplicación. El análisis se realizó mediante estadística descriptiva. Se consideró trastorno de ansiedad una puntuación igual o mayor de 16.

\section{| | | | | | | | | | | | | | | | | | | | | | | | | | | | | | | | | | | | | | | | | | | | | | | | | | | | | | | | | | | | | | | | | | | | | | | | | | | | | | | | | | | | | | | | | | | | | | | | | | | | | | | | | | | |}

\section{Resultados}

Se estudiaron 416 adolescentes con un promedio de edad de 14.3 años con un rango entre 12 a 17 años. De acuerdo al género, la muestra quedó distribuída con un ligero predominio del sexo masculino, con un $51.9 \%$ (216) y de $49.1 \%$ (200) para el sexo femenino. En cuanto al promedio de la puntuación obtenida en la escala de Hamilton fue de $11.6 \pm 0.7$ en un rango entre 0 y 38 puntos.
De los 416 adolescentes estudiados, 108 presentaron una puntuación $\geq 16$ con lo cual se considera la existencia de un cuadro ansioso de moderada intensidad que amerita tratamiento (no especificado ya que requiere valoración psiquiátrica). De éstos, 69 fueron mujeres y 39 hombres (63.8\% y $36.2 \%$ respectivamente). A partir de lo anterior se calculó una prevalencia de $25.96 \%$ de trastorno de ansiedad en nuestra población de estudio. 


\title{
Tabla I
}

\begin{abstract}
Adolescentes valorados con la escala de Hamilton, según promedio de edad, rango y distribución por género
\end{abstract}

\begin{tabular}{|ll|}
\hline $\mathrm{n}=416$ adolescentes & \\
\hline Edad (años) & $14.3(12$ a 17$)$ \\
\hline Género & Masculino $(51.9 \%)$ \\
& Femenino $(49.1 \%)$ \\
\hline
\end{tabular}

\section{Tabla 2}

Total de adolescentes con calificación en la escala de Hamilton $\geq$ 16, que califica como ansiedad de moderada intensidad, distribuida por género en el Municipio de Aguascalientes

\begin{tabular}{|l|l|}
\hline Género & $\begin{array}{l}\text { Adolescentes con calificación } \geq 16 \text { según } \\
\text { escala de Hamilton } \\
\mathrm{n}=108(25.9 \%)\end{array}$ \\
\hline Femenino & $69(63.8 \%)$ \\
\hline Masculino & $39(36.2 \%)$ \\
\hline
\end{tabular}

\section{Discusión}

Los datos de este estudio muestran una prevalencia de trastorno de ansiedad en los estudiantes de secundaria del municipio de Aguascalientes de un $25.9 \%$. En los trabajos publicados hasta la fecha, existe un rango amplio de la prevalencia del trastorno de ansiedad, que va desde el $9.19 \%$ al $48 \% 13$. Nuestros resultados se encuentran dentro de este rango (25.9\%) Esta prevalencia elevado puede estar asociada, entre otros factores, a que la mayoría de las secundarias incluídas, se localizaron en áreas con un nivel socioeconómico bajo. De tal manera que se puede especular que los adolescentes estudiados se desenvuelven en una estructura familiar difícil, en donde se puede observar deserción escolar y bajo rendimiento académico. Este entorno adverso puede ser trascendental en la formación de la personalidad de cada individuo y potencializa la predisposición que pudiese tener para desarrollar algún trastorno de ansiedad.

La presencia de ansiedad en la población adolescente estudiada, tuvo una distribución con predominio del género femenino sobre el masculino, como ya ha sido reportado por otros autores ${ }^{14}$. Este predominio puede influir otros fenómenos biológicos como el inicio de la vida reproductiva con la instauración de los ciclos menstruales.

Se ha descrito que el temperamento del adolescente con ansiedad se manifiesta por una actividad elevada, baja ritmicidad biológica e inhibición conductual, es decir, el trastorno de ansiedad puede dar lugar a un adolescente difícil, provocador de respuestas negativas hacia las personas que lo rodean; de tal manera que podemos especular que la prevalencia encontrada en este estudio puede ayudar a explicar el incremento observado en el consumo de alcohol y drogas ilícitas, en este sector de la 
población, con la finalidad de disipar este trastorno ${ }^{15,16}$.

Creemos importante continuar en esta línea de investigación para poder identificar los principales factores que tiene el medio en el que se desenvuelven los adolescentes de nuestro estado.

\section{Bibliografía}

1 Rynn MA, Riddle MA, Young PP, Kunz NR. Efficacy and safety of extended-release venlafaxine in the treatment of generalized anxiety disorder in children and adolescents: two placebo-controlled trials. Am J Psychiatry. 2007; 164(2):290-300.

2 Sakolsky D, Birmaher B. Pediatric anxiety disorders: management in primary care. Curr Opin Pediatr 2008;20(5):538-43

3 American Psychiatric Association; 2000 American Psychiatric Association. Revision Diagnostic and Statistical Manual of Mental Disorders 4th ed. Text. Washington, DC.

4 Twenge JM. The age of anxiety? Birth cohort change in anxiety and neuroticism, 1952-1993. J Pers Soc Psychol. 2000;79(6):1007-21.

5 Victor AM, Bernstein GA. Anxiety disorders and posttraumatic stress disorder update. Psychiatr Clin North Am. 2009;32(1):57-69.

6 Goodwin RD, Fergusson DM, Horwood LJ. Asthma and depressive and anxiety disorders among young persons in the community. Psychol Med. 2004; 34(8):1465-74.

7 Vazsonyi AT, Trejos-Castillo E, Huang L. Risky sexual behaviors, alcohol abuse, and drug abuse: a comparison of Eastern and Western European adolescents. J Adolesc Health. 2006;39(5):753.

8 Strine TW, Mokdad AH, Balluz LS, Gonzalez O, Crider R, Berry JT, Kroenke K.Depression and anxiety in the United States: findings from the 2006 Behavioral Risk Factor Surviellance System. Psychiatr Serv. 2008;59(12):1383-90

9 Freys T, Melzer D, Jenkins R, Brughan T. The distribution of the common mental disorders: social inequalites in Europe. Clin Practice and Epidemiology in Mental Health. 2005;1:14-26.
10 Toledos Castillo M. Atención de Adolescentes en los Servicios De Salud Mental del Hospital Hermilio Valdizán, Perú. Advances in Relational Mental Health. 2006;.5(2);1-37.

11 Caraveo Anduaga J. Cuestionario Breve De Tamizaje Y Diagnóstico De Problemas De Salud Mental En Niños Y Adolescentes: Algoritmos Para Síndromes Y Su Prevalencia En La Ciudad De México. Segunda Parte. Salud Mental. 2007;30(1): 48-55

12 Caraveo Anduaga J, Colmenares BE. Morbilidad psiquiátrica en la Ciudad de México: prevalencia y comorbilidad a lo largo de la vida; División de Investigaciones Epidemiológicas y Sociales. Revista Mexicana de Salud Mental. 1999: 62 - 67.

13 Tanja $M$, Ulrike $Z$, Jürgen $M$; Epidemiology of anxiety disorders. Department of Clinical Psychology and Psychotherapy, University of Basle, Switzerland; 2007; Epidemiology and Psychopharmacology. 2007:136-142.

14 Ozer EJ, Fernald LC, Roberts SC. Anxiety symptoms in rural Mexican adolescent: a social-ecological analysis. Soc Psychiatry Psychiart Epidemiol. 2008;43 (12):1014-23.

15 Medina-Mora ME, Borges G, Lara Muñoz C, Benjet C, Blanco Jaimes J, Fleiz Bautista C, Villatoro Velásquez J, Rojas Guiot E, Zambrano Ruíz J, Casanova Rodas L, Aguilar-Gaxiola S, Prevalencia De Trastornos Mentales y Uso De Servicios: Resultados De La Encuesta Nacional De Epidemiología Psiquiátrica en México

16 Jorge J. Caraveo-Anduaga, Eduardo Colmenares Bermúdez Los Trastornos Psiquiátricos y el abuso de sustancias en México: Panorama Epidemiológico.

17 Drentea P. Age, debt and anxiety. Health Soc. Behav. 2000, 41 (4): 437-50. 